\title{
Análisis bibliométrico de tesis doctorales españolas en lactancia materna, entre los años 1980-2015
}

Bibliometric analysis of Spanish doctoral theses on breastfeeding, between the years 1980-2015 Análise bibliométrica de teses de doutorado espanholas em aleitamento materno, entre os años 1980-2015

Ana García Rubioํㅜ Carmen Isabel Gómez García², Pilar Martínez Clares $^{3}$ y María Jesús Ruíz García ${ }^{4}$

${ }^{1}$ Escuela Internacional de Doctorado, Universidad de Murcia, Murcia, España ${ }^{2}$ Profesora Titular de Universidad. Perteneciente a clases pasivas. Facultad de Enfermería, Universidad de Murcia, Murcia, España

${ }^{3}$ Profesora Titular de Universidad. Dpto. de Métodos de Investigación y

Diagnóstico en Educación. Facultad de Educación, Universidad de Murcia, Murcia, España

${ }^{4}$ Profesora Titular de Universidad. Dpto. Enfermería. Facultad de Enfermería, Universidad de Murcia, Murcia, España

Cómo citar este artículo en edición digital: García Rubio, A., Gómez García, C.I., Martínez Clares, P., y RuIz García, M. ㅍ. (2019). Análisis bibliométrico de tesis doctorales españolas en lactancia materna, entre los años 1980- 2015.Cultura de los Cuidados (Edición digital), 23 (54). Recuperado de http://dx.doi.org/10.14198/cuid.2019.54.16

Correspondencia: Ana García Rubio. Cl José Antonio Camacho, 7, 30010, Murcia

Correo electrónico de contacto: ana.garcia7@um.es Recibido: 12/12/2018; Aprobado: 09/06/2019

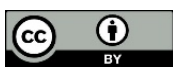

\section{ABSTRACT}

The current society inserted in a VUCA world (volatile, uncertain, complex and ambiguous) has produced vertiginous changes in relation to breastfeeding. That is why we consider it necessary to analyze the knowledge and scientific production carried out to date on this discipline. From this concern, this research emerges as an interdisciplinary study that aims to give priority to the protection, promotion and support of breastfeeding. The purpose is to investigate the growth and distribution of scientific documents through their analysis and evaluation, in order to gather the scientific production of researchers on breastfeeding. To this end, a sample of 155 Doctoral Theses published in Spanish Universities is used, with the common denominator of the word breastfeeding, in the period that goes from 1980 to 2015 . The methodology used is mixed, with qualitative data analysis, framed within a retrospective and descriptive analysis, as well as a quantitative analysis through bibliometric indicators. The aim is to offer a solid and reliable set of systematic, empirical and critical research processes and results, in order to present information on current and future research and innovation trends. 
Keywords: Breastfeeding, health sciences, nursing, bibliometric indicators, doctoral theses.

\section{RESUMO}

A sociedade atual inserida em um mundo da VUCA (volátil, incerto, complexo e ambíguo) produziu mudanças vertiginosas em relação ao aleitamento materno. Por isso, consideramos necessário analisar o conhecimento e a produção científica realizada até o momento nesta disciplina. A partir desta preocupação, esta pesquisa surge como um estudo interdisciplinar que visa dar prioridade à proteção, promoção e apoio da aleitamento materno. Objetivo é investigar o crescimento e a distribuição de documentos científicos por meio de sua análise e avaliação, a fim de coletar a produção científica de pesquisadores em aleitamento materno. Para tanto, utiliza-se uma amostra de 155 teses de doutorado publicadas em universidades espanholas, com o denominador comum da palavra amamentação, no período de 1980 a 2015. A metodologia utilizada é mista, com análise qualitativa dos dados, enquadrado em uma análise retrospectiva e descritiva, bem como uma análise quantitativa através de indicadores bibliométricos. $\mathrm{O}$ objetivo é oferecer um conjunto sólido e confiável de processos e resultados sistemáticos, empíricos e críticos de pesquisa, a fim de apresentar informações sobre tendências atuais e futuras de pesquisa e inovação.

Palavras chave: Aleitamento materno, ciências da saúde, enfermagem, indicadores bibliométricos, teses de doutorado.

\section{RESUMEN}

La sociedad actual inserta en un mundo VUCA (volátil, incierto, complejo y ambiguo) ha producido cambios vertiginosos en relación a la Lactancia
Materna (LM). Es por ello que consideramos preciso analizar el conocimiento y la producción científica efectuada hasta la fecha sobre esta disciplina. De esta inquietud, surge esta investigación como un estudio interdisciplinar que pretende dar prioridad a la protección, promoción $\mathrm{y}$ apoyo a la LM. La finalidad es investigar el crecimiento y la distribución de los documentos científicos a través de su análisis y evaluación, con el fin de recoger la producción científica de los investigadores en LM. Para ello se utiliza una muestra de 155 Tesis Doctorales publicadas en las Universidades Españolas, con el denominador común de la palabra clave lactancia materna, en el período comprendido entre los años 1980 a 2015. La metodología utilizada es mixta, con análisis de datos cualitativos, enmarcados dentro de un análisis retrospectivo y descriptivo, así como un análisis cuantitativo mediante indicadores bibliométricos. Se trata de ofrecer un conjunto sólido $y$ fiable de procesos y resultados sistemáticos, empíricos y críticos de investigación, a fin de exponer la información sobre las tendencias actuales y futuras de investigación e innovación.

Palabras clave: Lactancia materna, ciencias de la salud, enfermería, indicadores bibliométricos, tesis doctorales.

\section{INTRODUCIÓN}

El estudio de las tesis doctorales como objeto de análisis constituye un espejo donde se reflejan las líneas y tendencias de investigación de las universidades, es un medio idóneo para conocer la estructura social de la investigación, ya que permite analizar no sólo la evolución científica de una disciplina o área completa de estudio, 
sino también identificar cuáles son sus principales protagonistas y como se relacionan entre sí. (Repiso et al., 2011, p.157). El análisis de las publicaciones científicas constituye un eslabón fundamental dentro del proceso de investigación, convirtiéndose en una herramienta que nos permite calificar la calidad del proceso generador de conocimiento y el impacto de este proceso en el entorno. (Rueda- Clausen et al., 2005, p. 30).

La publicación y difusión de los resultados de cualquier investigación, al margen del área que se trate, constituye uno de los pilares de la ciencia; El acto de creación de la investigación científica estaría incompleto sin la publicación. Al ser ésta, la que facilita el nexo de unión, evaluación, y llegado el caso, la aceptación de la investigación y de sus resultados por parte de la comunidad científica. (Price, 1966). En este contexto, aplicar indicadores Bibliométricos, se constituye como uno de los procedimientos en la evaluación de la actividad investigadora.

La Bibliometría como disciplina científica se fundamenta en la búsqueda de comportamientos estadísticamente regulares a lo largo del tiempo, en los diferentes elementos relacionados con la producción y el consumo de información científica. Estas razones, justifican el interés de elaborar un estudio Bibliométrico capaz de proporcionar información exhaustiva de las tesis doctorales realizadas en las universidades españolas, en el período comprendido entre los años 1980 a 2015, que tengan como tópico de estudio la Lactancia Materna (LM). Previamente se ha comprobado que no existe ningún otro trabajo similar y se ha puesto de manifiesto el alto nivel que dichos estudios han conseguido dentro de los programas universitarios españoles, así como las bondades de esta práctica en la salud y educación de las mujeres y los lactantes.

La investigación que nos ocupa pretende realizar una revisión sistemática, crítica e integradora de las tesis doctorales existentes en este campo y que con un carácter interdisciplinar han ofrecido una comprensión más profunda del tema y del significado y alcance de la LM, dando prioridad a la protección, promoción $\mathrm{y}$ apoyo a la misma. A través de revisión sistemática bibliográfica y el análisis de contenido de las tesis se ha evidenciado cuáles son las fortalezas y las limitaciones $\mathrm{u}$ obstáculos que impiden avanzar o frenan el cambio que se ha producido a lo largo de la historia en LM. Hablar sobre LM es hablar de la historia del ser humano, como proceso biológico, es una actividad cultural que afectará a las mujeres independientemente de sus creencias o clase social. El análisis teórico de los conocimientos actuales que desde la historia, sociología, piscología, biología molecular y anatomía, se están produciendo sobre los beneficios de la LM, se verá completado con este estudio, para redefinir gran parte del conocimiento que tenemos en LM, surgiendo la siguiente pregunta de investigación ¿Qué se ha investigado sobre LM en las universidades españolas?

\section{Objetivos de investigación}

1. Analizar las tesis doctorales publicadas en universidades españolas entre los años académicos 1980/81 y 2014/15, con un muestreo de ciento cincuenta y cinco tesis doctorales extraídas de bases de datos digitales.

2. Exponer de manera cuantitativa los resultados, por medio del uso de un conjunto de indicadores bibliométricos.

3. Determinar la productividad científica 
de los investigadores en LM, producidas por un autor, departamento, institución o disciplina, a través de la aplicación de la llamada Ley de Lotka.

4. Describir las relaciones existentes entre los productores científicos que han determinado a través de una red social con el estudio de Indicadores de Colaboración.

5. Exponer la información recogida sobre las tendencias de investigación, metodologías empleadas, innovación y actualidad, comprobando el estado actual de la investigación.

\section{MATERIAL Y MÉTODO}

El presente trabajo es un análisis de la estructura productiva de la investigación española catalogada dentro de la Lactancia Materna, a través de los datos extraídos de las tesis doctorales en España, en el período 1980/2015. Utilizando la base de datos bibliográfica TESEO, Tesis Doctorales en Red (TDR), Tesis digitalizadas de libre acceso de la biblioteca Virtual Miguel de Cervantes, DIALNET, y por último, tesis digitales de REBIUN (Red de bibliotecas universitaria).

\section{Muestra}

Para ello se ha utilizado una muestra de 155 tesis doctorales publicadas en universidades españolas entre el período de 1980 a 2015. Con el fin de recoger la producción científica de los investigadores en LM.

\section{Procedimiento y análisis}

Para la realización de este proyecto, la información se ha tratado con el software ofimático de Microsoft Office, usándose sus aplicaciones específicas Word 2011 y Excel 2011. Como herramienta para la gestión de referencias bibliográficas, se ha usado Mendeley Desktop, con la finalidad de identificar, capturar, etiquetar y clasificar las tesis doctorales. Se ha realizado un registro individualizado de todos los investigadores, cuando los datos bibliográficos eran insuficientes para determinar todos los datos necesarios para catalogar a los investigadores, se ha realizado una búsqueda documental a través del motor de búsqueda Google, intentando acceder a la referencia completa de la tesis doctoral. Si aun así no era posible acceder a todos los datos, hemos intentado ponernos en contacto vía correo electrónico a través de las instituciones colaboradoras.

Una vez recuperados los documentos de las bases de datos analizadas, se han ordenado para incluirlos a través de Microsoft Excel 2011 en una base de datos propia, dándole forma y contenido a través de las siguientes variables:

TABLA 1

\section{TESIS DOCTORAL}

Título Tesis

Autor

Tutores y/o Directores de Tesis

Fecha de Defensa

Universidad

Departamento 
Nombres miembros del Tribunal

Mención Europea

Línea de Trabajo

Tipología de Tesis

Tipo de Estudio

FUENTE: Elaboración propia

\section{INDICADORES BIBLIOMÉTRICOS EMPLEADOS}

Los indicadores bibliométricos empleados para el análisis de los datos han consistido en:

\section{Indicadores de productividad, forma $y$ contenido}

Este conjunto de indicadores es el medio más simple de cuantificación de la cantidad de investigación que produce un agente investigador, y permite observar la evolución temporal del agregado (Cabezas, 2013, p.46). Se obtienen a partir del recuento de las publicaciones científicas. Se puede medir la productividad científica de un autor, departamento o grupo de investigación, una institución, disciplina o un país durante un período de tiempo determinado, entre los que destacamos los que a continuación se relatan:

* Autores más productivos: En este sentido, se ha calculado este indicador encaminado a identificar el porcentaje de miembros más activos en investigación en LM.

* Instituciones, Universidad, Facultad y Departamentos más productivos: Entre las bondades de los estudios bibliométricos podemos definir los patrones, tendencias y regularidades de la producción científica por instituciones, universidades, facultades o departamentos. Así como hacer comparaciones y ponderaciones entre ellas y sus temáticas.
* Años más productivos: Otro análisis de interés, tiene que ver con los años más productivos desde 1980 a 2015, permitiendo comparar la labor de los investigadores y grupos de investigación según la capacidad que tienen para influir en el desarrollo de nuevos artículos y la generación de nuevo conocimiento.

* Líneas de investigación: Centrándonos en las líneas de investigación llevadas a cabo en los artículos en LM, a través de un análisis del contenido muestra su grado de especialización. Para su categorización, se han agrupado según la clasificación utilizada en este estudio, analizando la cantidad de documentos publicados en cada clase temática, midiendo de esta manera la distribución por frecuencias de la producción temática.

* Tipos de estudio: Los indicadores de contenido se refieren al estudio de los ejes centrales en una publicación, su metodología se puede analizar a través de las palabras más significativas en los títulos, resúmenes, descriptores o el material y método empleado en el estudio.

\section{Indicadores de colaboración}

Los indicadores de colaboración, aplicados al estudio de las publicaciones científicas permiten conocer el grado de cooperación que un agente científico presenta en su actividad productiva. En este estudio, el 
grado de colaboración se mide desde una perspectiva individual, calibrando el número medio de autores por trabajo, como desde una perspectiva institucional.

* Índice de coautoría: Este indicador se utiliza para determinar la actividad y cooperación científica.

* Autoría por sexo: El estudio de la igualdad entre mujeres y hombres es uno de los ámbitos de atención prioritarios para gobiernos $y$ administraciones públicas y su tratamiento es imprescindible en el ámbito universitario. Por ello, se ha introducido la variable sexo como categoría de análisis, para analizar la igualdad o desigualdad de género.

* Colaboración internacional: Refleja las tesis doctorales en los que aparece como firmante una institución no española junto a otros autores españoles, así como tesis doctorales con mención internacional.

\section{ANÁLISIS ESTADÍSTICO}

El análisis de los diferentes datos se realiza a través de las medidas estadísticas de la media y de la desviación típica. El cálculo de los indicadores se realizó con MS Access 2011, mientras que para el análisis estadístico se ha usado el paquete informático SPSS en su versión 20.0.

\section{RESULTADOS Y DISCUSIÓN}

Se presentan los principales resultados de este trabajo desagregados por indicadores bibliométricos y para el corte temporal de 1980 a 2015. Indicadores de recursos humanos, producción y colaboración científica, actividad, autoría y finalmente obsolescencia, le dan forma y contenido a este estudio.

\section{INDICADORES \\ DE \\ PRODUCTIVIDAD, FORMA Y CONTENIDO}

A través de estos indicadores se ha estudiado la producción científica de los autores de tesis doctorales, sus directores y los miembros del tribunal que participaron en las diferentes defensas de tesis. Así como, las instituciones desde las que se gestaron dichos estudios y el año en el que sucedió. Sin olvidar los tipos de estudio y líneas de investigación que han dado forma $y$ contenido a estas tesis doctorales.

\section{Directores de tesis doctorales más productivos}

En estos 35 años estudiados, como ya se ha mencionado con anterioridad, se han identificado un total de 203 directores o codirectores de tesis doctorales. Siendo importante mencionar que un gran número de ellos solamente ha dirigido una tesis dentro de esta área. A continuación, se presentan aquellos directores que han dirigido más de una tesis. En 1996 y desde la Universidad de Granada nos encontramos con la primera tesis, de las 3 dirigidas por el Dr. Bayes García. Destacar que en las dos primeras décadas estudiadas, era muy frecuente contar con solo un director de tesis. Continuando con la Universidad de Granada, la Dra. Campoy Folgoso, dirige en 2002 su primera tesis codirigida junta al Dr. Bayes García, constatándose a través de esta variable la agregación de este tándem como grupo de investigación desde la Universidad de Granada. También desde la Medicina y más concretamente desde la Universidad Autónoma de Barcelona, nos encontramos con la Dra. Castellote Baragalló, codirigiendo 3 tesis doctorales desde la primera tesis que nos encontramos censada en nuestra base datos en 2002. Desde esta Universidad también nos 
encontramos con la Dra. López Sabater, que junto a la Dra. Castellote Baragalló, dirigen otra tesis desde la Facultad de Farmacia, la primera de ellas en 2006.

Cambiando de disciplina desde la Facultad de Enfermería de la Universidad de Murcia una figura relevante y precursora del estudio de la LM desde la enfermería, la Dra. Gómez García dirige en 2006 la primera tesis de la que hay constancia en las bases de datos consultadas. La tesis doctoral de la Dra. Morales Gil, desde la Facultad de Ciencias de la Salud de la Universidad de Málaga, realizando su aportación sobre la "Valoración del empleo de metodología NANDA-NOC-NIC en Lactancia materna". La Dra. Gómez García ha dirigido entre otras tesis, como la llevada a cabo en 2011 desde la Universidad de Murcia y uno de los pocos estudios cualitativos desde la disciplina enfermera, "Un estudio cualitativo sobre Lactancia Materna prolongada en un grupo de madres de Murcia" llevado a cabo por la Dra. Ruíz García.

$\mathrm{Si}$ nos centramos ahora en otras figuras relevantes, desde la Universidad de Zaragoza destacamos al Dr. Rodríguez Martínez y la Dra. Samper Villagrasa, pareja de investigadores que desde 2011, tal y como consta en las bases de datos consultadas han dirigido varias tesis conjuntas. Constatándose de nuevo una red de escuelas científicas y redes académicas desde esta Universidad que participan en la producción conjunta en LM.

\section{Tribunales de tesis doctorales más productivos}

El número total de profesionales que han participado en tribunales de tesis doctorales referidas a la LM en el período estudiado es de 553. A continuación relatamos una muestra de los que han estado en más de un tribunal de tesis, siendo un total de 89. Así, se pone de manifiesto la importancia de diferentes académicos que ocupan puestos destacados durante este período.

La Dra. Ballester Añon desde la Universidad de Alicante del Departamento de Enfermería Comunitaria, Medicina Preventiva y Salud pública e Historia de la Ciencia ha estado presente en hasta en 3 ocasiones como Presidenta de tribunales de Tesis en su Universidad.

Desde el Departamento de Pediatría de la Facultad de Medicina de la Universidad de Granada, tenemos de nuevo a la Dra. Campoy Folgoso, que además de dirigir varias tesis doctorales, ha sido testigo de varias defensas de tesis desde 1993 a 2007, tales como, Granada, Barcelona y Málaga. También desde ésta universidad nos encontramos al Dr. Gómez Capilla desde el Departamento de Bioquímica y Bilogía Molecular, que de 1993 a 2014 también a participado en 5 tribunales de tesis. Continuando con la Universidad de Granada, nos encontramos al Dr. Molina Font, profesor titular de la Universidad de Granada con plaza vinculada al Servicio Andaluz de Salud, que ha participado hasta en 7 tribunales de tesis doctorales, desde 1996 hasta 2014 en Universidades tales como, Granada y Barcelona. El Dr. Narbona López, Catedrático del Departamento de Pediatría de la Facultad de Medicina, también de la Universidad de Granada, nos consta que desde 1988 hasta 1998 ha participado hasta en 4 tribunales de tesis.

Desde la isla de la "eterna primavera" destacamos a la Dra. Díaz Gómez, profesora titular de la Universidad de La Laguna del departamento de Enfermería, participando como nos consta en las bases de datos consultadas, desde 2006 hasta 2012 en varios tribunales de tesis, tanto en las Universidades de Málaga como de Murcia. 
Trasladándonos al otro extremo del país, desde el Hospital Universitario Marqués de Valdecilla (Santander), más concretamente el Servicio de Pediatría, destacamos al Dr. García Fuentes, que desde 1989 hasta 2003, ha participado en 5 tribunales de tesis en universidades como Zaragoza, País Vasco, Cantabria o Granada. También desde la Universidad de Santiago de Compostela, desde el Departamento de Ciencias forenses, Anatomía Patológica, Ginecología y Obstetricia y Pediatría, destacamos al Dr. Tojo Sierra, con su participación hasta en 4 tribunales desde 1989 a 2008 en universidades de País Vasco, Santiago de Compostela y Granada.

Continuando en nuestro ámbito, la Enfermería, destacar al Dr. Linares Abad, desde la Universidad de Jaén y el Departamento de Enfermería, participando en diferentes tribunales de tesis como vocal desde 2009 hasta 2013 en las universidades de Alicante, Granada y Málaga. Desde la Unidad Docente de Matronas de la Región de Murcia, la Dra. Martínez Roche ha participado en hasta 4 tribunales de tesis doctorales desde 2006 hasta 2015, pasando desde la Universidad de Murcia hasta Alicante y Universidad de Málaga. Y es desde ésta última universidad desde donde destacamos la figura de la Dra. Morales Gil, profesora titular de Universidad en la Facultad de Ciencias de la Salud de la Universidad de Málaga. Presenciando hasta 4 defensas de tesis doctorales desde 2011 hasta 2014, en las universidades de Murcia y Málaga.

\section{Instituciones más productivas}

Las tesis producidas se han defendido en 30 universidades diferentes, estamos por tanto ante una herramienta de análisis científico de amplia difusión en la universidad española. No obstante, un número reducido de tesis concentran la mayoría de tesis defendidas: Autónoma de Barcelona, Autónoma de Madrid, Complutense de Madrid, Alicante, Barcelona, Granada, Málaga y Murcia. Convirtiéndose en los principales productores de trabajos en LM y el auténticos faros que han guiado la proliferación de estudios en esta temática.

En cuanto a Facultades de lectura, se pone de manifiesto el carácter médico que tiene la LM, concentrándose fundamentalmente entre las Facultades de Medicina y Ciencias de la Salud, siendo bastante inferior en otros campos del conocimiento. No obstante, son las Facultades de Medicina las que adquieren mayor protagonismo, situándose en segundo lugar las Facultades de Ciencias de la Salud. Aludimos al bajo número de tesis defendidas en las Facultades de Enfermería al hecho de que muchas de ellas se encuentren adscritas dentro de Facultades de Ciencias de la Salud como Departamentos de Enfermería. Resultado que se corrobora en el estudio de tesis defendidas por departamentos, donde se puede observar, que el Departamento de Enfermería junto al del Pediatría y Pediatría, Ginecología y Obstetricia encabezan el ranking de Departamentos donde se han defendido más tesis doctorales.

\section{Años más productivos}

El análisis de la evolución productiva evidencia un crecimiento del número de tesis leídas, dicho crecimiento se va produciendo al mismo ritmo y en los mismos plazos temporales que la extensión de la enseñanza universitaria, el crecimiento en el número de alumnos y la inversión en recursos humanos que esta conllevó (Delgado et al., 2006, p. 499). Así en la década de 1980 a 1990 nos encontramos con 15 tesis doctorales leídas, número que se duplica en 
la siguiente década de 1991 a 2000 con 32 tesis leídas en universidades españolas. Investigaciones del más alto nivel que va en un progresivo aumento, continuando con la siguiente década de 2001 a 2011 nos encontramos con 41 tesis doctorales. Pero es especialmente en el último quinquenio estudiado, en el cual se va a producir la eclosión definitiva, ya que en la mitad de tiempo que el anteriormente analizado se han leído 67 tesis doctorales. Esta cifra puede considerase como un signo de interés por este tema que en la actualidad aún permanece sobre la LM en España.

\section{Líneas de investigación}

Las líneas de investigación estudiadas se refieren principalmente a temas relacionados a analizar ya sea bien desde una metodología cualitativa como cuantitativa aspectos de la LM, seguido de líneas de investigación cuya finalidad era la promoción de la LM. Siendo las revisiones históricas aquellas áreas en las que menos profundizan las tesis doctorales.

\section{Tipos de estudio}

Las metodologías que han prevalecido han sido la Cualitativa Descriptiva con líneas de investigación que analizan la situación actual de la LM o fomentan la promoción de la misma. Y los de metodología Cuantitativa Analítica de Cohortes o Casos - controles, que han analizado desde epidemiología de la LM, su composición o los factores que influyen en su permanencia o abandono.

\section{INDICADORES DE COLABORACIÓN}

Estos indicadores, permiten conocer el grado de cooperación que los protagonistas estudiados presentan en su actividad productiva a través de estos indicadores, el grado de colaboración se mide tanto desde una perspectiva individual, calibrando que autores han producido tesis en LM, como una perspectiva institucional, caracterizando la colaboración a la hora de dirigir tesis, como su presencia en tribunales. No menos importante es conocer la colaboración internacional o la autoría por sexo.

\section{Índice de coautoría}

En el siguiente apartado se ha considerado cruzar dos indicadores anteriormente presentados, para situar a cualquier director en relación con la comunidad a la que pertenece. Para ello, se ha establecido una genealogía de autores de tesis doctorales en LM, dirección de tesis doctoral y afiliación a tribunal. Y posteriormente se ha completado con una red de coparticipación de los diferentes académicos como directores de tesis y participación en tribunales, ya que no todos nos han constado como autores de tesis en LM en las bases de datos consultadas.

Por tanto, si analizamos las estructuras reticulares de coparticipación, podemos observar como casi todos ellos han estado presentes en evaluaciones de otras universidades estrechamente relacionadas, presentando en muchos casos además la característica común de tener una relación bidireccional. Otras variables nos permiten estudiar cómo se han interrelacionado los investigadores como directores de tesis doctorales junto a su presencia en tribunales tanto de sus universidades como en otras universidades. Mostrándose activos en cuanto a dirección de tesis, liderando proyectos de investigación así como creando lo que hoy en día se llama "la ciencia de las redes". Se puede estudiar su estructura social a nivel micro y comprobar cómo es un patrón que se repite en la mayor parte de todos los autores. 
Tras estudiar las escuelas científicas o redes sociales que se han ido creando, se puede confirmar una unión de las universidades de la zona centro de España con el triada entre Universidad de Alcalá de Henares, Complutense de Madrid y Autónoma de Madrid guiado por los Dr. Nogales Espert y Dr. Álvarez De Los Heros. Destacar la presencia del Dr. Tojo Sierra que ha estado presente en tribunales de Universidad de Granada y País Vasco. Continuando con la Universidad de Granada, destacar la escuela científica entre las universidades de Granada y Barcelona, dominadas por las figuras, Dra. Campoy Folgoso, Dra. López Sabater y Dr. Molina Font. Otra escuela científica es la formada por la Dra. Lozano de La Torre y el Dr. Sanjurjo Crespo a través de encuentros bidireccionales entre las universidades de Cantabria y País Vasco.

Bajando hacía el sur, nace la escuela científica formada por la Dra. Gómez García perteneciente a la Universidad de Murcia, que conecta los tribunales de las universidades de Málaga a través de la Dra. Morales Gil y Alicante y Miguel Hernández de Elche a través de los doctores Cortés Castell, Juste Ruíz y Rizo Baeza. A esta escuela científica se une la Dra. Díaz Gómez de la Universidad de La Laguna. Quedando establecida una red de investigadores expertos en LM.

\section{Autoría por sexo}

El objetivo de este indicador es analizar la representación las diferencias de género en LM, comprobando si existen diferencias de sexo en estas investigaciones de alto nivel. El $69,03 \%$ de los trabajos son firmados por mujeres, es decir, 107 de los 155 analizados han sido producidos por mujeres, siendo casi el doble que los firmados por hombres, confirmando la presencia de mujeres en el campo de la producción de tesis doctorales en LM.

\section{Colaboración Internacional}

Tan solo 3 tesis doctorales constan en las bases de datos consultadas, con mención internacional que se han llevado a cabo en el terreno de la LM, aportando 1,93\%. Dichas tesis doctorales pertenecen al sistema universitario catalán, 3 universidades que se encuentran en grandes áreas metropolitanas, Barcelona y Tarragona. No se ha obviado que de las 3 tesis doctorales con mención internacional, ninguna ha sido realizada desde la enfermería. Habitualmente, solo se hace eco de las grandes investigaciones, de los avances tecnológicos, de la robotización o de nuevos hospitales, invitándonos a reflexionar, ¿por qué no podemos comenzar a visibilizar la enfermería española nuestras investigaciones en cuidados a nivel internacional? Con la llegada de la convergencia del Espacio Europeo de Educación superior (EEES), tenemos la oportunidad de un espacio abierto en el que no existen obstáculos de movilidad de estudiantes, con garantía de calidad.

\section{CONCLUSIONES, LIMITACIONES Y PROSPECTIVAS}

Si tuviéramos que poner fecha y escenario al resurgimiento de la disciplina enfermera, sería tras la creación del EEES, la implantación del Grado de enfermería y la posibilidad de realización de másteres académicos oficiales. Se abrieron las puertas a los profesionales enfermeros para desarrollar una carrera investigadora, que incluiría entre sus hechos más relevantes la defensa de una tesis doctoral.

Hemos constatado una evidente heterogeneidad tanto en la elaboración de 
tesis, como dirección, así como presencia en tribunales. Poniéndose de manifiesto a través de las escuelas científicas o redes académicas que se han ido creando a través de una colaboración bilateral entre centros a nivel nacional. En este sentido, la propuesta es lograr que diferentes instituciones encuentren la congruencia y el punto de equilibrio entre los investigadores, haciendo uso de investigaciones multidisciplinares, para realizar nuevas aportaciones desde diferentes perspectivas al estudio de la LM. Aludimos el crecimiento producido a finales de los años 90 y principios del nuevo milenio en lo que a producción científica en LM se refiere, debido a las medidas gubernamentales impulsadas para promover la LM, reconociéndose oficialmente la LM como una prioridad.

El estudio de las unidades de análisis ayuda a dilucidar las líneas de investigación más definidas como es, el análisis y promoción de la LM bajo las metodologías más empleadas, Cualitativa Descriptiva y Cuantitativa Analítica de Cohortes o Casoscontroles. En cuanto a diferencias de género en LM, el $69,03 \%$ de los trabajos son firmados por mujeres. Un estudio llevado a cabo por López Gómez en 2016, destaca que se aprecia una tendencia a estar más representadas las mujeres en la autoría de la producción científica. Aunque a pesar de esto, los últimos datos del Ministerio de Sanidad aluden a que solo un $20 \%$ del total de Catedráticos está formado por mujeres. No se ha encontrado predominio de colaboración internacional, dicha dimensión es una de las principales características de la ciencia actual, reflejándose su peso cada vez más en los evidentes cambios en cuanto a producción del conocimiento se refiere.

En cuanto a limitaciones destacar que las bases de datos no recogen el total de tesis producidas en España y nos gustaría recordar que "Sin datos no hay visibilidad y sin visibilidad no hay prioridad" (Huyer y Westholm, 2002, p. 84).

\section{REFERENCIAS BIBLIOGRÁFICAS}

- Arias, F. G. (2017). Obsolescencia de las referencias citadas: un mito académico persistente en la investigación universitaria. E-Ciencias de la Información, 7(1), 1-14.

- Berchid-Martínez, F., Herrero-Hahn, R., y HuesoMontoro, C. (2017). Producción científica en enfermería transcultural en el periodo 2007-2014. Cultura de los Cuidados (Edición digital), 21(49), pp. 2345. Recuperado de http://dx.doi.org/10.14198/cuid.2017.49.13.

- Buela-Casal, G., Bermúdez, M.P., Sierra, J.C., GuillénRiquelme, A. y Quevedo-Blasco, R. (2012). Productividad y eficiencia en investigación por comunidades autónomas españolas según la financiación. Revista Iberoamericana de Psicología y Salud. 6(1), pp. 1-10. Recuperado de http://www.sciencedirect.com/science/article/pii/S21 71206915700013\#bib0005.

- Cabezas Clavijo, A. (2013). Estudio Bibliométrico de la producción científico, actividad y colaboración científicas en grupos de investigación: el caso de la Universidad de Murcia. (Tesis doctoral). Granada: Universidad de Granada.

- Curiel-Marín, E., y Fernández-Cano, A. (2015). Análisis cienciométrico de tesis doctorales españolas en didáctica de las ciencias sociales (1976-2012). Revista Española de Documentación Científica. 38(4), e110.

- Delgado López-Cózar, E., Torres-Salinas, D., Jiménez-Contreras, E. \& Ruiz-Pérez, R. (2006). Análisis bibliométrico y de redes sociales aplicado a las tesis bibliométricas defendidas en España (19762002): temas, escuelas científicas y redes académicas. Revista Española de Documentación Científica, vol. 29 (4), 493-524.

- Huyer, S. y Westholm, G. (2002). Toolkit in Gender Indicators in Engineering, Science and Technology. UNESCO/GAB. Paris: Unesco.

- López Gómez, E. (2016). Análisis de las tesis doctorales sobre tutoría: aproximación bibliométrica y tendencias temáticas. Rev. gen. inf. doc. 26(1), 147$164 . \quad$ Recuperado de http://revistas.ucm.es/index.php/RGID/article/view/ $\underline{53047 .}$.

- Ministerio de Educación, Cultura Deporte. (2018). Datos y cifras del sistema universitario español. Curso 


\section{Cultura de los Cuidados}

2016-17. Madrid: Secretaría General Técnica. Subdirección General de Documentación y Publicaciones. Recuperado de http://estadisticas.mecd.gob.es/EducaJaxiPx/Datos.ht m?path=/Universitaria/Alumnado/Estadistica/20162 017/1GradoCiclo/CapituloI//10/\&file=mat_1.px\&type $=$ pcaxis.

- Price, D. J. S. (1963). Little science, big science. Nueva York: Columbia University Press.

- Repiso, R., Torres, D. \& Delgado, E. (2011). Análisis bibliométrico y redes sociales en tesis doctorales españolas sobre televisión $(1976$ - 2007). Comunicar, 37, 151-159.

- Rueda-Clausen Gómez, C. F, Villa-Roel Gutiérrez, C. \& Rueda-Clausen Pinzón, C. E. (2005). Indicadores bibliométricos: Origen, contradicción y nuevas propuestas. Revista MedUNAB, 8(1), 29-36. 